\title{
Topological Fiber Entropy for Linear Flows on Vector Bundles
}

\author{
Fritz Colonius • Luiz A.B. San Martin • \\ Adriano J. da Silva
}

\begin{abstract}
For linear flows on vector bundles, it is shown that the topological entropy of lower dimensional subspaces in the fibers is determined by the Morse spectrum over chain recurrent components of the induced flows on Grassmann bundles.
\end{abstract}

Keywords Topological entropy $\cdot$ Linear flows $\cdot$ Bilinear control systems

Mathematics Subject Classification 2010 37B40 · 37H15

\section{Introduction}

The purpose of these notes is to compute topological entropies for linear flows on vector bundles. For linear flows, which have a noncompact state space, the entropies have to be defined with respect to compact, in general noninvariant, subsets $K$ of initial values. Classical results for linear maps by R. Bowen [4] show that the unstable eigenvalues determine the topological entropy. Since linear flows are nonautonomous dynamical systems given by a linear cocycle over a base flow, one will expect that Lyapunov exponents play an analogous role here when entropy in the base space is neglected. However, things are complicated by two facts as follows: In contrast to the measure theoretic situation, the topological situation leads to "many" Lyapunov exponents, which may be captured in the

F. Colonius ( $\square)$

Institut für Mathematik, Universität Augsburg, 86135 Augsburg, Germany

e-mail: fritz.colonius@math.uni-augsburg.de

L. A. B. San Martin

Instituto de Matemática, Universidade Estadual de Campinas, Cx. Postal 6065 13.081-970 Campinas, SP, Brasil

e-mail: smartin@ime.unicamp.br

A. J. da Silva

Institut für Mathematik, Universität Augsburg, 86159 Augsburg, Germany

e-mail: smartin@ime.unicamp.br 
Morse spectrum (see Colonius/Kliemann [7]) consisting of compact intervals associated to the chain recurrent components in the projective bundle. Furthermore, since volume growth plays a key role, the topological entropy depends critically on the considered sets of initial values. It turns out that the Morse spectrum corresponding to the chain recurrent components in the associated Grassmann bundles comes into play (see Colonius and Kliemann [9], Braga Barros and San Martin [5], San Martin and Seco [21]). Our main result (Theorem 5.2) relates the topological entropy of a $k$-dimensional subspace $V$ of a fiber to the Morse spectrum in the Grassmann bundle obtained by projection to the unstable subbundle.

Topological entropy for nonautonomous dynamical systems has found some interest in the literature. For example, Kolyada and Snoha [14] define an entropy for nonautonomous dynamical systems. A main motivation for their work, which is restricted to compact spaces, comes from skew product systems; see also Zhang and Chen [23] for a recent contribution. A classical result by Abramov and Rokhlin (see, e.g., Petersen [17]) shows that the metric entropy of a skew product flow is the sum of the entropy of the base flow and the fiber entropy. For skew product flows with compact fibers, Bogenschütz [3] proved a variational principle relating metric fiber entropy and topological fiber entropy. Instead, we consider noncompact (linear) fibers and linear flows. Furthermore, we concentrate on the fiber entropy, since in control systems, which provide a major motivation for our work, the topological entropy of the base space is infinite. It is also worth to note that the fiber entropy considered in the present paper depends on the metric. We refer to Handel and Kitchens [11] and Patrão [15] who discuss related questions in the general context of topological entropy in noncompact spaces.

In Section 2, we briefly recall the finest Morse decomposition of linear flows in Grassmann bundles and the main properties of the associated Morse spectrum. Furthermore, some notions from multilinear algebra are recalled. Section 3 introduces fiber entropy for linear flows on vector bundles and presents an estimate from below. Section 4 uses the Oseledets theorem to establish that the fiber entropy is determined by the corresponding interval of the Morse spectrum on Grassmannian bundles, at least for all points in the base space for which the Oseledets theorem holds. Here, also the special case of bilinear control systems is discussed.

\section{Linear Flows on Vector Bundles}

In this section, facts on linear flows on vector bundles, on the chain transitive components of their induced flows on Grassmannians, and on the associated Morse spectra are recalled. We also fix some notation from multilinear algebra. For the general theory of continuous flows on compact metric spaces, we refer to the recent book by Alongi and Nelson [1], and for the definition of vector bundles $\pi: \mathcal{V} \rightarrow B$, we refer to Karoubi [13] (or [8, Appendix B]). Locally, they are the product of an open subset of a metric space $B$ with a finite dimensional Hilbert space $H$. We always assume that the base space $B$ is compact. A continuous bilinear form on $\mathcal{V}$ is constructed by adding the inner products in the local charts with appropriate weights taking into account the distance in the base space $([8$, p. 535]). All norms obtained in this way are equivalent. A linear flow $\Phi$ on a vector bundle $\pi: \mathcal{V} \rightarrow B$ is a (continuous) flow $\Phi$ on $\mathcal{V}$ such that for all $\alpha \in \mathbb{R}$ and $v_{1}, v_{2} \in \mathcal{V}$ with $\pi\left(v_{1}\right)=\pi\left(v_{2}\right)$ and $t \in \mathbb{R}$, one has

$$
\pi\left(\Phi\left(t, v_{1}\right)\right)=\pi\left(\Phi\left(t, v_{2}\right)\right), \Phi\left(t, \alpha\left(v_{1}+v_{2}\right)\right)=\alpha \Phi\left(t, v_{1}\right)+\alpha \Phi\left(t, v_{2}\right)
$$


Where notationally convenient, we write instead of $\Phi(t, v)$ either $\Phi_{t}(v)$ or $\Phi(t) v$. The flow $\Phi$ induces a flow $\theta$ on the base space $B$ (corresponding to transport of the fibers) and flows on the Grassmann bundles $\mathbb{G}_{k} \mathcal{V}$ and, in particular, on the projective bundle $\mathbb{P} \mathcal{V}=\mathbb{G}_{1} \mathcal{V}$. For ease of notation, we also denote these induced flows by $\Phi$. A metric in $\mathbb{P V}$ is constructed using the metric on the projective space for $H$ given by

$$
\mathrm{d}\left(\mathbb{P} v, \mathbb{P} v^{\prime}\right):=\min \left(\frac{v}{\|v\|}-\frac{v^{\prime}}{\left\|v^{\prime}\right\|}, \frac{v}{\|v\|}+\frac{v^{\prime}}{\left\|v^{\prime}\right\|}\right),
$$

where $v, v^{\prime}$ are elements of a fiber $\mathcal{V}_{b}$ which are not in the zero section $Z \subset \mathcal{V}$ (for distances in fiber bundles, see also the general construction of [16], Section 3.3). The cocycle associated with $\Phi$ consists of the following family of maps on the fibers

$$
\varphi(t, b): \mathcal{V}_{b} \rightarrow \mathcal{V}_{\theta_{t} b}: v \mapsto \Phi(t, v) \text { for }(t, b) \in \mathbb{R} \times B,
$$

satisfying the cocycle property

$$
\varphi\left(s, \theta_{t} b\right) \varphi(t, b) v=\varphi(s+t, b) v \text { for } s, t \in \mathbb{R}, b \in B, \text { and } v \in \mathcal{V}_{b} .
$$

The following theorem goes back to Selgrade [22]; see also Salamon and Zehnder [20], Colonius and Kliemann [8].

Theorem 2.1 (Selgrade) Let $\Phi$ be a linear flow on a vector bundle $\pi: \mathcal{V} \rightarrow B$ with chain transitive flow on the base space $B$. Then the chain recurrent set of the induced flow $\mathbb{P} \Phi$ on the projective bundle $\mathbb{P} \mathcal{V}$ has finitely many, linearly ordered components $\left\{\mathcal{M}_{1}, \ldots, \mathcal{M}_{l}\right\}$, and $1 \leq l \leq d:=\operatorname{dim} \mathcal{V}_{b}, b \in B$. Every chain recurrent component $\mathcal{M}_{i}$ defines an invariant subbundle of $\mathcal{V}$, called Selgrade bundle, via

$$
\mathcal{V}_{i}=\mathbb{P}^{-1}\left(\mathcal{M}_{i}\right)=\left\{v \in \mathcal{V}, v \notin Z \text { implies } \mathbb{P} v \in \mathcal{M}_{i}\right\}
$$

and the following decomposition into a Whitney sum holds:

$$
\mathcal{V}=\mathcal{V}_{1} \oplus \cdots \oplus \mathcal{V}_{l}
$$

For points $v \in \mathcal{V}$ which are not in the zero section $Z$ in $\mathcal{V}$, the Lyapunov exponent or exponential growth rate of the corresponding trajectory is given by

$$
\lambda(v):=\limsup _{t \rightarrow \infty} \frac{1}{t} \log \left\|\Phi_{t} v\right\|
$$

and the Lyapunov spectrum $\Sigma_{L y}$ of the linear flow $\Phi$ is the set of all Lyapunov exponents $\Sigma_{L y}=\{\lambda(v), v \in \mathcal{V} \backslash Z\}$. The concept of Morse spectrum is defined via $(\varepsilon, T)$-chains in the projective bundle. Recall that for $\varepsilon, T>0$, an $(\varepsilon, T)$-chain $\zeta$ in $\mathbb{P V}$ of $\Phi$ is given by $n \in \mathbb{N}$, $T_{0}, \ldots, T_{n-1} \geq T$, and $p_{0}, \ldots, p_{n}$ in $\mathbb{P} \mathcal{V}$ with $d\left(\Phi\left(T_{i}, p_{i}\right), p_{i+1}\right)<\varepsilon$ for $i=0, \ldots, n-1$. Define the finite time exponential growth rate of such a chain $\zeta$ (or "chain exponent") by

$$
\lambda(\zeta)=\left(\sum_{i=0}^{n-1} T_{i}\right)^{-1} \sum_{i=0}^{n-1}\left(\log \left\|\Phi\left(T_{i}, v_{i}\right)\right\|-\log \left\|v_{i}\right\|\right),
$$

where $v_{i} \in \mathbb{P}^{-1}\left(p_{i}\right)$. Then the Morse spectrum over a chain recurrent component $\mathcal{M}$ is

$$
\Sigma_{M o}(\mathcal{M}, \Phi)=\left\{\begin{array}{c}
\lambda \in \mathbb{R}, \text { there are } \varepsilon^{j} \rightarrow 0, T^{j} \rightarrow \infty \text { and }\left(\varepsilon^{j}, T^{j}\right) \text {-chains } \\
\zeta^{j} \text { in } \mathcal{M} \text { with } \lambda\left(\zeta^{j}\right) \rightarrow \lambda \text { as } j \rightarrow \infty
\end{array}\right\} .
$$

The Morse spectrum $\Sigma_{M o}(\Phi)$ of $\Phi$ is defined as the union of the Morse spectra over the chain recurrent components in $\mathbb{P} \mathcal{V}$. The main properties of the Morse spectrum are collected in the following theorem [8]. 
Theorem 2.2 Let $\Phi$ be a linear flow on a vector bundle $\pi: \mathcal{V} \rightarrow B$ with chain transitive flow on the base space $B$. Then

$$
\Sigma_{L y}(\Phi) \subset \Sigma_{M o}(\Phi)=\bigcup_{i=1}^{l} \Sigma_{M o}\left(\mathcal{M}_{i}, \Phi\right),
$$

where the $\mathcal{M}_{i}$ are the chain recurrent components of the projective flow $\mathbb{P} \Phi$. Furthermore, for every $i$,

$$
\Sigma_{M o}\left(\mathcal{M}_{i}, \Phi\right)=\left[\kappa^{*}\left(\mathcal{M}_{i}\right), \kappa\left(\mathcal{M}_{i}\right)\right]
$$

with $\kappa^{*}\left(\mathcal{M}_{i}\right)<\kappa^{*}\left(\mathcal{M}_{j}\right)$ and $\kappa\left(\mathcal{M}_{i}\right)<\kappa\left(\mathcal{M}_{j}\right)$ for $i<j$; the boundary points $\kappa^{*}\left(\mathcal{M}_{i}\right), \kappa\left(\mathcal{M}_{i}\right)$ are obtained as limits for $t \rightarrow \pm \infty$ for all points in the base space $B$ outside of sets with measure zero for certain ergodic measures.

We also note that, for a chain transitive base space, the Morse spectrum coincides with the Sacker-Sell spectrum [19]; one obtains the exponentially dichotomous subbundles provided by Sacker-Sell theory by taking in the decomposition (2) the sum of all subbundles with intersecting Morse spectral intervals.

For the volume growth and its relation to the chain recurrent components in the Grassmannian bundles $\mathbb{G}_{k} \mathcal{V}$, it will be helpful to keep in mind the following facts from multilinear algebra. For a (real) Hilbert space $H$ of dimension $d$ with scalar product denoted by $\langle\cdot, \cdot\rangle$, let $\omega: H^{k} \rightarrow \mathbb{R}$ be an alternating $k$-linear map. For $\left(x_{1}, \ldots, x_{d}\right) \in H^{d}$, consider $\left(y_{1}, \ldots, y_{k}\right) \in H^{k}$ with $y_{i}=\sum_{j=1}^{d} b_{i j} x_{j}$. Then one computes

$$
\omega\left(y_{1}, \ldots, y_{k}\right)=\sum_{j_{1}<\ldots<j_{k}} \operatorname{det} B_{j_{1} \ldots j_{k}} \omega\left(x_{j_{1}}, \ldots, x_{j_{k}}\right),
$$

where $B_{j_{1} \ldots j_{k}}$ is the $k \times k$-matrix obtained from the columns $j_{1}, \ldots, j_{k}$ of $B=\left(b_{i j}\right)$ and summation is over all ordered $k$-combinations of $\{1, \ldots, d\}$. The map $\omega$ defines a $k$-dimensional volume for $H$. For $1 \leq k \leq d$, the $k$-fold alternating product $\wedge{ }^{k} H=H \wedge \cdots \wedge H$ is defined as the vector space with an alternating $k$-linear map $\omega_{\wedge}: H^{k} \rightarrow \wedge^{k} H:\left(x_{1}, \ldots, x_{k}\right) \mapsto$ $x_{1} \wedge \cdots \wedge x_{k}$ with (i) if $\left(x_{1}, \ldots, x_{d}\right)$ is a basis of $H$, then

$$
\left\{x_{j_{1}} \wedge \cdots \wedge x_{j_{k}} \mid 1 \leq j_{1} \leq \cdots \leq j_{k} \leq d\right\}
$$

is a basis of $\bigwedge^{k} H$, and (ii) if $\varphi: H^{k} \rightarrow \mathbb{R}$ is an alternating $k$-linear map, then there is a unique linear map $\theta: \wedge^{k} H \rightarrow \mathbb{R}$ with $\varphi=\theta \circ \omega_{\wedge}$.

The elements of the form $x_{1} \wedge \cdots \wedge x_{k}$ are called indecomposable and $\left(\wedge^{k} H\right)_{\text {Ind }} \subset$ $\bigwedge^{k} H$ is the subset of all indecomposable elements. If $e_{1}, \ldots, e_{d}$ is an orthonormal basis of $H$, then $\omega\left(e_{j_{1}}, \ldots, e_{j_{k}}\right)=1,1 \leq j_{1}<\cdots<j_{k} \leq d$, defines via (4) an alternating bilinear form. The exterior product is the bilinear form determined by

$\bigwedge^{j} H \times \wedge^{k} H \rightarrow \wedge^{j+k} H:\left(y_{1} \wedge \cdots \wedge y_{j}, z_{1} \wedge \cdots \wedge z_{k}\right) \mapsto y_{1} \wedge \cdots \wedge y_{k} \wedge z_{1} \wedge \cdots \wedge z_{k}$.

For an orthonormal basis $e_{1}, \ldots, e_{d}$ of $H$, one obtains a basis of $\bigwedge^{k} H$ by

$$
\left\{e_{j_{1}} \wedge \cdots \wedge e_{j_{k}} \mid 1 \leq j_{1} \leq \cdots \leq j_{k} \leq d\right\}
$$


By linear extension, this also induces an inner product $\langle\cdot, \cdot\rangle$ on $\bigwedge^{k} H$ by making the basis above orthonormal. The parallelepiped spanned by a $k$-tuple of linearly independent vectors in $H$, or $k$-parallelepiped, is

$$
\operatorname{co}\left\{\sum_{j=1}^{l} x_{i_{j}} \mid i_{j} \neq i_{j^{\prime}} \text { for } j \neq j^{\prime} \text { and } i_{1}, \ldots, i_{l} \in\{1, \ldots, l\}, l \in\{0,1, \ldots, k\}\right\} \text {. }
$$

For two such $k$-tuples $\left(x_{1}, \ldots, x_{k}\right),\left(y_{1}, \ldots, y_{k}\right)$, one has

$$
x_{1} \wedge \cdots \wedge x_{k}=y_{1} \wedge \cdots \wedge y_{k}
$$

if and only if $\left(x_{1}, \ldots, x_{k}\right),\left(y_{1}, \ldots, y_{k}\right)$ span the same subspace and the parallelepipeds $P$ spanned by them have the same volume given by $\operatorname{vol}_{k}(P)=\left\|x_{1} \wedge \cdots \wedge x_{k}\right\|$.

Hence, we can identify the set $\mathbb{G}_{k} H$ of $k$-dimensional subspaces in $H$ with the equivalence classes of indecomposable elements $x_{1} \wedge \cdots \wedge x_{k}$ in $\wedge{ }^{k} H$ differing only by a nonzero factor. In other words, we can identify $\mathbb{G}_{k} H$ with the subset $\mathbb{P}\left(\bigwedge^{k} H\right)_{\text {Ind }}$ of projective space $\mathbb{P}\left(\bigwedge^{k} H\right)$. It is not hard to see that under the metric (1), this subset is compact, and in the following we use this metric for $\mathbb{G}_{k} H$.

We endow $H$ with the following inner product which is adapted to a decomposition into linear spaces $L_{j}, 1 \leq j \leq l$ : for each $j$, one has a basis $e_{1}^{j}, \ldots, e_{d_{j}}^{j}$ of $L_{j}$ which is orthonormal with respect to the given inner product. Define

$$
\left\langle e_{i_{1}}^{j_{1}}, e_{i_{2}}^{j_{2}}\right\rangle=\left\{\begin{array}{l}
0 \text { for } j_{1} \neq j_{2} \text { or } i_{1} \neq i_{2} \\
1 \text { for } j_{1}=j_{2} \text { and } i_{1}=i_{2}
\end{array}\right.
$$

The chain recurrent components on Grassmannians (and more generally on flag manifolds) are expressed as fixed point sets of diagonalizable matrices, viewed as maps of the Grassmannians. If $h$ is a diagonalizable linear map of $H$, then a point $\mathbb{P} v$ of $\mathbb{P}$ is fixed by $h$ if and only if $v$ is an eigenvector of $h$. Hence, the connected components of the fixed point set fix $\mathbb{P}_{\mathbb{P}}(h)$ of $h$ in $\mathbb{P}$ are the sets $\mathbb{P} W$ with $W \subset H$ an eigenspace of $h$.

The same way, the fixed points of $h$ in $\mathbb{G}_{k} H$ are spanned by the decomposable vectors $x_{1} \wedge \cdots \wedge x_{k}$ that are eigenvectors of $\wedge^{k} h$, that is,

$$
\wedge^{k} h\left(x_{1} \wedge \cdots \wedge x_{k}\right)=h x_{1} \wedge \cdots \wedge h x_{k}=\lambda\left(x_{1} \wedge \cdots \wedge h x_{k}\right) .
$$

Hence, the fixed point set fix $\mathbb{G}_{k} H(h)$ of $h$ in $\mathbb{G}_{k} H$ is the $\mathbb{P}\left(W \cap\left(\bigwedge^{k} \mathcal{V}\right)_{\text {Ind }}\right)$ with $W$ running through the eigenspaces of $\bigwedge^{k} h$. The connected components of fix $\mathbb{G}_{k} H(h)$ has a simple description in terms of the eigenspaces $W_{1}, \ldots, W_{l}$ of $h$ : Let $d_{i}=\operatorname{dim} W_{i}, i=1, \ldots, l$, and take integers $\left(k_{1}, k_{2}, \ldots, k_{l}\right)$ with $k_{1}+k_{2}+\cdots+k_{l}=k$ and $0 \leq k_{i} \leq d_{i}$. Then

$$
W_{k_{1}, \ldots, k_{l}}^{k}=\left\{V \in \mathbb{G}_{k} H: \operatorname{dim}\left(V \cap W_{i}\right)=k_{i}\right\}
$$

is a connected component of fix $\mathbb{G}_{k} H(h)$, and these sets exhaust the components. Below, we also write

$$
W_{k_{1}, \ldots, k_{l}}^{k}=\mathbb{G}_{k_{1}} W_{1} \oplus \cdots \oplus \mathbb{G}_{k_{l}} W_{l}
$$

with the interpretation that on the right hand side, we have the sum of arbitrary $k_{i}$ dimensional subspaces of $W_{i}$.

All this can be extended in a standard way to bundles: One constructs the $k$-fold alternating product bundle $\bigwedge^{k} \mathcal{V}$ and the subset $\left(\bigwedge^{k} \mathcal{V}\right)_{\text {Ind }}$ of indecomposable elements as well as the Grassmann bundle $\mathbb{G}_{k} \mathcal{V}$, with inner products on $\mathcal{V}$ adapted to (2), by performing these constructions on every chart $H \times U$, where $U$ is open in the base $B$. One obtains a continuous bilinear form on the vector bundle which is equivalent to the original one. This does not 
change the chain transitive sets or the Lyapunov exponents. A linear flow $\Phi$ on $\mathcal{V}$ induces a linear flow on $\wedge^{k} \mathcal{V}$ if we define $v_{1} \wedge \cdots \wedge v_{k} \rightarrow \Phi_{t} v_{1} \wedge \cdots \wedge \Phi_{t} v_{k}, v_{i} \in \mathcal{V}_{b}$, on the indecomposable elements and then extend by linearity. Then

$$
\left\|\Phi\left(t, v_{1}\right) \wedge \cdots \wedge \Phi\left(t, v_{k}\right)\right\|
$$

is the volume of the parallelepiped spanned by the images under the map $\Phi(t, \cdot)$ of $v_{1}, \ldots, v_{k}$.

Again, we can identify the compact set $\mathbb{P}\left(\bigwedge^{k} \mathcal{V}\right)_{\text {Ind }}$ with $\mathbb{G}_{k} \mathcal{V}$. This yields a (locally trivial) fiber bundle $\left(\bigwedge^{k} \mathcal{V}\right)_{\text {Ind }} \rightarrow \mathbb{G}_{k} \mathcal{V}$. The induced flow is fiber preserving.

The following theorem holds by Braga Barros and San Martin [5, Theorem 10.1] and Patrão and San Martin [16, Theorem 10.1], combined with the above description of the components of fixed point set fix $\mathbb{G}_{k} H(h)$.

Theorem 2.3 Let $\Phi$ be a linear flow on a vector bundle $\pi: \mathcal{V} \rightarrow B$ with chain transitive base space $B$ and dimension $d$. Let $\mathcal{V}_{i}$ with dimension $d_{i}, i=1, \ldots, l$, be the Selgrade bundles. Define for $1 \leq k \leq d$ the index set

$$
I(k):=\left\{\left(k_{1}, k_{2}, \ldots, k_{l}\right), k_{1}+k_{2}+\cdots+k_{l}=k \text { and } 0 \leq k_{i} \leq d_{i}\right\} .
$$

Then the chain recurrent components in the Grassmann bundle $\mathbb{G}_{k} \mathcal{V} \rightarrow B$ are given by the sets

$$
\mathcal{M}_{k_{1}, \ldots, k_{l}}^{k}=\mathbb{G}_{k_{1}} \mathcal{V}_{1} \oplus \cdots \oplus \mathbb{G}_{k_{l}} \mathcal{V}_{l},\left(k_{1}, \ldots, k_{l}\right) \in I(k),
$$

with the interpretation that on the right hand side, we have in every fiber $\mathcal{V}_{b}$ the sum of arbitrary $k_{i}$-dimensional subspaces of $\mathcal{V}_{i, b}$. Hence, the elements of $\mathcal{M}_{k_{1}, \ldots, k_{l}}^{k}$ are the $k$-dimensional subbundles $\mathcal{W}$ with $\operatorname{dim}\left(\mathcal{W}_{b} \cap \mathcal{V}_{i, b}\right)=k_{i}, i=1, \ldots, l$, or, equivalently, with

$$
\mathcal{W}=\bigoplus_{i=1}^{l}\left[\mathcal{W} \cap \mathcal{V}_{i}\right]
$$

We turn to determine the exponential growth rates of volumes. The associated spectrum is defined as follows. For $\varepsilon, T>0$, consider an $(\varepsilon, T)$-chain $\zeta$ of $\Phi$ in $\mathbb{G}_{k} \mathcal{V}$ given by

$$
n \in \mathbb{N}, T_{0}, \ldots, T_{n-1} \geq T, V_{0}, \ldots, V_{n} \in \mathbb{G}_{k} \mathcal{V}
$$

with $d\left(\Phi\left(T_{i}, V_{i}\right), V_{i+1}\right)<\varepsilon$ for $i=0, \ldots, n-1$. Identify the subspace $V_{i}$ and $v_{i 1} \wedge \ldots \wedge v_{i k}$ where $\left\|v_{i 1} \wedge \cdots \wedge v_{i k}\right\|=1$. Let the exponential growth rate of $\zeta$ be

$$
\lambda(\zeta):=\left(\sum_{i=0}^{n-1} T_{i}\right)^{-1}\left(\sum_{i=0}^{n-1} \log \left\|\Phi\left(T^{i}, v_{i 1}\right) \wedge \cdots \wedge \Phi\left(T_{i}, v_{i k}\right)\right\|\right)
$$

and define the Morse spectrum over a chain recurrent component by

$$
\Sigma_{M o}\left(\mathcal{M}_{k_{1}, \ldots, k_{l}}^{k}, \Phi\right):=\left\{\begin{array}{c}
\lambda \in \mathbb{R}, \text { there are } \varepsilon^{j} \rightarrow 0, T^{j} \rightarrow \infty \text { and } \\
\left(\varepsilon^{j}, T^{j}\right) \text {-chains } \zeta^{j} \text { in } \mathcal{M}_{k_{1}, \ldots, k_{l}}^{k} \text { with } \Lambda\left(\zeta^{j}\right) \rightarrow \lambda
\end{array}\right\} .
$$

Furthermore, the Morse spectrum of the linear flow on the Grassmann bundle $\mathbb{G}_{k} \mathcal{V}$ is defined by taking the union over all chain recurrent components in $\mathbb{G}_{k} \mathcal{V}$. The following theorem is a consequence of [9, Theorem 9].

Theorem 2.4 For a chain recurrent component $\mathcal{M}_{k_{1}, \ldots, k_{l}}^{k}$ in the Grassmann bundle $\mathbb{G}_{k} \mathcal{V}$, the associated Morse spectrum is a compact interval

$$
\Sigma_{M o}\left(\mathcal{M}_{k_{1}, \ldots, k_{l}}^{k}, \Phi\right)=\left[\kappa^{*}\left(\mathcal{M}_{k_{1}, \ldots, k_{l}}^{k}\right), \kappa\left(\mathcal{M}_{k_{1}, \ldots, k_{l}}^{k}\right)\right] .
$$


All limit points for $t \rightarrow \infty$ of growth rates along a trajectory in the chain recurrent component are in this interval. The boundary points are obtained as limits for $t \rightarrow \pm \infty$ for all points in the base space $B$ outside of sets with measure zero for certain ergodic measures.

The following lemma is a consequence of [6, Theorem 2.7].

Lemma 2.5 For the fiber bundle $\pi:\left(\bigwedge^{k} \mathcal{V}\right)_{\text {Ind }} \rightarrow \mathbb{G}_{k} \mathcal{V}$, consider the continuous function $f:\left(\wedge^{k} \mathcal{V}\right)_{\text {Ind }} \rightarrow \mathbb{R}$ given by $f\left(v_{1} \wedge \cdots \wedge v_{k}\right):=\log \left\|v_{1} \wedge \cdots \wedge v_{k}\right\|$. Then we have that for $\left\|v_{1} \wedge \cdots \wedge v_{k}\right\|=1$

$$
\limsup _{t \rightarrow+\infty} \frac{1}{t} f\left(\Phi_{t}\left(v_{1} \wedge \cdots \wedge v_{k}\right)\right) \in \Sigma_{M o}\left(\mathcal{M}_{k_{1}, \ldots, k_{l}}^{k}\right)
$$

where $\mathcal{M}_{k_{1}, \ldots, k_{l}}^{k}$ is the Morse set that contains the omega limit of the point $\pi\left(v_{1} \wedge \cdots \wedge v_{k}\right)$ for the induced flow in $\mathbb{G}_{k} \mathcal{V}$.

Proof Since $f\left(\Phi_{t}\left(v_{1} \wedge \cdots \wedge v_{k}\right)\right)-f\left(v_{1} \wedge \cdots \wedge v_{k}\right)$ only depends on the fiber and the limit superior is uniformly bounded over the base space, the function $f$ satisfies the two basic assumptions (2) and (3) in [6], cp. also [6, Remark 2.1].

\section{Fiber Entropy}

In this section, we define the topological fiber entropy of linear flows and derive some of its properties.

Let $\Phi$ be a linear flow on a vector bundle $\mathcal{V} \rightarrow B$ with base flow $\theta_{t}(b)$ and cocycle $\varphi(t, b), t \in \mathbb{R}, b \in B$. Consider a compact subset $K$ contained in a $k$-dimensional subspace $V$ of a fiber $\mathcal{V}_{b}$.

A set $R$ in $V$ is called $(T, \varepsilon, K$; $\Phi)$-spanning if for every $v \in K$ there is $w \in R$, such that for all $t \in[0, T]$ one has

$$
\|\varphi(t, b) v-\varphi(t, b) w\|=\left\|\Phi_{t} v-\Phi_{t} w\right\| \leq \varepsilon,
$$

where the norms are taken in the fibers $\mathcal{V}_{b \cdot t}$. Denoted by $r(T, \varepsilon, K ; \Phi)$ is the minimal cardinality of a $(T, \varepsilon, K ; \Phi)$-spanning set.

Definition 3.1 With the notation above, the topological fiber entropy of $\Phi$ with respect to $K$ is defined by

$$
h(\varepsilon, K ; \Phi):=\limsup _{T \rightarrow \infty} \frac{1}{T} \log r(T, \varepsilon, K ; \Phi), h(K ; \Phi):=\lim _{\varepsilon \searrow 0} h(\varepsilon, K ; \Phi) .
$$

and the fiber entropy of $V$ is

$$
h(V, \Phi)=\sup _{K \subset V}\{h(K, \Phi)\} .
$$

If the base space is a point, then we have a linear flow on a vector space and the entropy is determined by the eigenvalues of the (linear) time-one map; cf. Bowen [4] or Walters [24, Theorem 8.14]. The definition above does not look at the topological entropy of the linear flow $\Phi$ on the vector bundle $\mathcal{V}$, since the behavior in the base space $B$ is not taken into account. Instead, we only study the behavior with respect to the fibers. It is immediate from the definition that $h(K ; \Phi) \leq h\left(K^{\prime} ; \Phi\right)$ for $K \subset K^{\prime}$. 
A set $S \subset K$ is called $(T, \varepsilon, K$; $\Phi)$-separated if for all $v, w \in S$ with $v \neq w$ there is $t \in[0, T]$ with $\|\varphi(t, b) v-\varphi(t, b) w\|>\varepsilon$. Denoted by $s(n, \varepsilon, K ; \Phi)$ is the maximal cardinality of a $(T, \varepsilon, K ; \Phi)$-separated set. It is easily seen that the topological entropy can also be defined via separated sets

$$
h(V ; \Phi):=\sup _{K \subset V}\left\{\lim _{\varepsilon \searrow 0} \limsup _{T \rightarrow \infty} \frac{1}{T} \log s(T, \varepsilon, K ; \Phi)\right\} .
$$

This follows, since a maximal $(T, \varepsilon, K ; \Phi)$-separated set is also $(T, \varepsilon, K ; \Phi)$-spanning, which implies $s(T, \varepsilon, K ; \Phi) \geq r(T, \varepsilon, K ; \Phi)$; furthermore, using the triangle inequality, one sees that $s(T, \varepsilon, K ; \Phi) \leq r(T, 2 \varepsilon, K ; \Phi)$.

In order to estimate the fiber entropy, the following technical lemma is needed.

Lemma 3.2 Let $K$ be a compact subset contained in a $k$-dimensional subspace $V$ of a fiber $\mathcal{V}_{b}$. Then the fiber entropy with respect to $K$ is invariant under translations and multiplication by scalars, that is,

$$
h(K, \Phi)=h(K+v, \Phi)=h(\alpha K, \Phi),
$$

for all $v \in V$ and all $\alpha \neq 0$.

Proof Let $R$ be a $(T, \varepsilon, K, \Phi)$-spanning set with cardinality $r(T, \varepsilon, K, \Phi)$ and consider the set $R^{\prime}=R+v$. Then for all $w^{\prime} \in K+v$, there is $w \in K$ and $u \in R$ such that $w^{\prime}=w+v$ and $\|\varphi(t, b) w-\varphi(t, b) u\|<\varepsilon, t \in[0, T]$. By the linearity of the flow, we can conclude that $\left\|\varphi(t, b) w^{\prime}-\varphi(t, b) u^{\prime}\right\|<\varepsilon$, where $u^{\prime}=u+v \in R^{\prime}$. Therefore, $R^{\prime}$ is a $(T, \varepsilon, K+v, \Phi)$-spanning set and $r(T, \varepsilon, K+v, \Phi) \leq r(T, \varepsilon, K, \Phi)$. In the same way, taking a $(T, \varepsilon, K+v, \Phi)$-spanning set, we obtain that

$$
r(T, \varepsilon, K+v, \Phi) \geq r(T, \varepsilon, K, \Phi),
$$

and consequently, we have the equality $h(K, \Phi)=h(K+v, \Phi)$. Similar arguments show the equality $h(K, \Phi)=h(\alpha K, \Phi)$.

By Lemma 3.2 we just need to calculate $h(K, \Phi)$ for $K$ being a $k$-parallelepiped in $V$, since in this case $h(K, \Phi)=h(V, \Phi)$. For the further analysis the following elementary properties of the fiber entropy will be needed.

Lemma 3.3 Consider a decomposition of $\mathcal{V}$ into measurable invariant subbundles $\mathcal{V}=\mathcal{W}_{1} \oplus \mathcal{W}_{2}$ valid over all $b$ in a subset $B_{0} \subset B$. Denote the corresponding projections by $\pi_{1,2}: \mathcal{V} \rightarrow \mathcal{W}_{1,2}$. Then for every compact set $K \subset \mathcal{V}_{b}, b \in B_{0}$, the fiber entropy with respect to $K$ and to $\pi_{1,2} K$ for the restrictions $\Phi_{1,2}: \mathcal{W}_{1,2} \rightarrow \mathcal{W}_{1,2}$ of $\Phi$ satisfies

$$
h(K, \Phi) \leq h\left(\pi_{1} K, \Phi_{1}\right)+h\left(\pi_{2} K, \Phi_{2}\right) \text { and } h(K, \Phi) \geq h\left(\pi_{1,2} K, \Phi_{1,2}\right) .
$$

Proof Fix $\varepsilon, T>0$ and consider for $i=1,2$ minimal $\left(T, \varepsilon, \pi_{i} K, \Phi_{i}\right)$-spanning sets $\hat{R}_{i} \subset \pi_{i} K$ with cardinality $r\left(T, \varepsilon, \pi_{i} K ; \Phi_{i}\right)$. Then there are subsets $R_{i} \subset K$ with the same cardinality such that $\hat{R}_{i}=\pi_{i}\left(R_{i}\right)$. Every element $v \in K$ can be written as $v=$ $\pi_{1} v \oplus \pi_{2} v$. Using the spanning property of $\hat{R}_{i}$ for the restrictions $\Phi_{i}$, one finds that $R_{1} \cup$ $R_{2}$ is $(\varepsilon, T, K ; \Phi)$-spanning. For $T \rightarrow \infty$ and then $\varepsilon \rightarrow 0$, the first assertion follows. For the second assertion, observe that a minimal $(T, \varepsilon, K, \Phi)$-spanning set projects to a $\left(T,|| \pi_{1,2} \| \varepsilon, \pi_{1} K, \Phi_{1}\right)$-spanning set. 


\section{Lower Bounds}

The lower bounds for the fiber entropy to be given in this section are based in the following estimate in terms of the Morse spectrum.

Proposition 4.1 Let $\Phi$ be a linear flow on a vector bundle $\mathcal{V}$. Consider a $k$-dimensional vector space $V \subset \mathcal{V}_{b}$. Then the topological entropy of $V$ is bounded below by the lower bound of the Morse spectrum of $\mathcal{M}_{k_{1}, \ldots, k_{l}}^{k}$

$$
h(V, \Phi) \geq \kappa^{*}\left(\mathcal{M}_{k_{1}, \ldots, k_{l}}^{k}\right),
$$

where $\mathcal{M}_{k_{1}, \ldots, k_{l}}^{k}$ is the Morse set that contains the omega limit of the point $V$ for the induced flow on $\mathbb{G}_{k} \mathcal{V}$.

Proof Let $K$ be a $k$-parallelepiped in $V$ spanned by $v_{1}, \ldots, v_{k} \in V$. Then for $t>0$, the $k$-dimensional volume of $\Phi_{t}(K)$ satisfies

$$
\operatorname{vol}_{k}\left(\Phi_{t}(K)\right)=\left\|\Phi_{t} v_{1} \wedge \cdots \wedge \Phi_{t} v_{k}\right\| .
$$

Let $\varepsilon>0, T>0$, and consider a $(T, \varepsilon, K ; \Phi)$-spanning set $R$ of minimal cardinality $r(T, \varepsilon, K$; $\Phi)$. Then (by the definition of spanning sets) the set $\Phi_{T}(K)$ is contained in the union of $r(T, \varepsilon, K ; \Phi)$ balls $B\left(w_{j}, \varepsilon\right)$ of radius $\varepsilon$,

$$
B\left(w_{j}, \varepsilon\right)=\left\{v \in \mathcal{V}_{b \cdot T} \mid\left\|v-w_{j}\right\|<\varepsilon\right\} .
$$

Each such ball has volume bounded by $c(2 \varepsilon)^{d}$, where $c>0$ is a constant. Thus

$$
\operatorname{vol}_{k}\left(\Phi_{T}(K)\right) \leq r(T, \varepsilon, K ; \Phi) \cdot c(2 \varepsilon)^{d} .
$$

This yields

$$
\log r(T, \varepsilon, K ; \Phi) \geq \log \left\|\Phi_{T} v_{1} \wedge \cdots \wedge \Phi_{T} v_{k}\right\|-\log \left[c(2 \varepsilon)^{d}\right],
$$

and hence by Lemma 2.5

$$
\begin{aligned}
& \limsup _{T \rightarrow \infty} \frac{1}{T} \log r(T, \varepsilon, K, \Phi) \\
& \geq \limsup _{T \rightarrow \infty} \frac{1}{T} \log \left\|\Phi_{T} v_{1} \wedge \cdots \wedge \Phi_{T} v_{k}\right\| \in \Sigma_{M o}\left(\mathcal{M}_{k_{1}, \ldots, k_{l}}^{k}\right) .
\end{aligned}
$$

For $\varepsilon \rightarrow 0$, one obtains the assertion.

From now on, we assume that $0 \notin \Sigma_{M o}(\Phi)$. This means that,

$$
\mathcal{V}=\mathcal{V}^{+} \oplus \mathcal{V}^{-}
$$

with $\Sigma_{M o}\left(\mathcal{V}^{+}\right) \subset(0,+\infty)$ and $\Sigma_{M o}\left(\mathcal{V}^{-}\right) \subset(-\infty, 0)$, where $\mathcal{V}^{+}$is the sum of the Selgrade bundles $\mathcal{V}_{i}$ with Morse spectrum contained in $(0,+\infty)$ and $\mathcal{V}^{-}$the sum of Selgrade bundles $\mathcal{V}_{i}$ with Morse spectrum contained in $(-\infty, 0)$.

Note that for $k$-dimensional vector space $V \subset \mathcal{V}_{b}^{-}$, we have that $h(V, \Phi)=0$, since for any compact set $K \subset V$, the minimal cardinality of a $(T, \varepsilon, K, \Phi)$ remains bounded as $T$ goes to infinity. Since we are interested in what happens in the unstable part, we will always assume that the projection of the vector space $V$ to the positive part $\mathcal{V}_{b}^{+}$has positive dimension. 
In the following, we denote by $\pi^{ \pm}$the projection of $\mathcal{V}$ to $\mathcal{V}^{ \pm}$. Furthermore, let $\mathcal{V}_{i}^{+}:=\mathcal{V}_{i} \cap \mathcal{V}^{+}$and, for a chain transitive set $\mathcal{M}_{k_{1}, \ldots, k_{l}}^{k}$, we define $\pi^{+} \mathcal{M}_{k_{1}, \ldots, k_{l}}^{k}$ as the set of all subbundles obtained by projecting the subbundles to $\mathcal{V}^{+}$. It is convenient to write

$$
k_{i}^{+}=\left\{\begin{aligned}
k_{i} & \text { if } \Sigma_{M o}\left(\mathcal{V}_{i}\right) \subset(0,+\infty) \\
0 & \text { if } \Sigma_{M o}\left(\mathcal{V}_{i}\right) \subset(-\infty, 0)
\end{aligned} \text { and } k^{+}:=k_{1}^{+}+\ldots+k_{l}^{+} .\right.
$$

Thus $k^{+}=\operatorname{dim} \mathcal{V}^{+}$and $k_{i}^{+}=0$ iff $\mathcal{V}_{i} \subset \mathcal{V}^{-}$. The following proposition, which describes what happens to the chain recurrent components in the Grassmannians when we apply $\pi^{+}$, is an immediate consequence of the definitions and Theorem 2.3.

Proposition 4.2 For elements $\mathcal{W}$ in a chain recurrent component $\mathcal{M}_{k_{1}, \ldots, k_{l}}^{k}$ in the Grassmann bundle $\mathbb{G}_{k} \mathcal{V}$, let

$$
\pi^{+} \mathcal{W}:=\bigoplus\left[\mathcal{W} \cap \mathcal{V}_{i}^{+}\right]
$$

Then the set

$$
\pi^{+} \mathcal{M}_{k_{1}, \ldots, k_{l}}^{k}:=\left\{\pi^{+} \mathcal{W} \mid \mathcal{W} \in \mathcal{M}_{k_{1}, \ldots, k_{l}}^{k}\right\}=\mathcal{M}_{k_{1}^{+}, \ldots, k_{l}^{+}}^{k^{+}}
$$

is a chain recurrent component in $\mathbb{G}_{k^{+}} \mathcal{V}^{+}$. For a $k$-dimensional parallelepiped $K$ in a fiber $\mathcal{W}_{b}$ of $\mathcal{W} \in \mathcal{M}_{k_{1}, \ldots, k_{l}}^{k}$ spanned by vectors in $\mathcal{V}_{i, b}, i=1, \ldots, l$, the set $\pi^{+} K$ is a $k^{+}$-dimensional parallelepiped in $\pi^{+} \mathcal{W}_{b}$.

Lemma 4.3 For the decomposition (8) of $\mathcal{V}$ into the stable and unstable invariant subbundles $\mathcal{V}^{+}$and $\mathcal{V}^{-}$, the topological entropy with respect to a compact set $K \subset \mathcal{V}_{b}$ satisfies

$$
h(K, \Phi)=h\left(\pi^{+} K, \Phi^{+}\right) .
$$

Proof In view of Lemma 3.3, we only have to show that $h\left(\pi^{-} K, \Phi^{-}\right)=0$. This follows, since the minimal cardinality of a $\left(T, \varepsilon, \pi^{-} K ; \Phi^{-}\right)$-spanning set remains bounded for $T \rightarrow$ $\infty$.

Now, we combine Lemma 3.3 with the lower bound of Proposition 4.1 to get the following sharper lower bound for the fiber entropy.

Theorem 4.4 Let $\Phi$ be a linear flow on a vector bundle $\mathcal{V}$ with $0 \notin \Sigma_{M o}(\Phi)$. Consider a $k$-dimensional vector space $V \subset \mathcal{V}_{b}$. Then the topological entropy of $V$ is bounded below by the lower bound of the Morse spectrum of $\mathcal{M}_{k_{1}^{+}, \ldots, k_{l}^{+}}^{k^{+}}$

$$
h(V, \Phi) \geq \kappa^{*}\left(\mathcal{M}_{k_{1}^{+}, \ldots, k_{l}^{+}}^{k^{+}}\right)
$$

where $\mathcal{M}_{k_{1}^{+}, \ldots, k_{l}^{+}}^{k^{+}}$is the Morse set that contains the omega limit of the point $\pi^{+}(V)$ for the induced flow on $\mathbb{G}_{k^{+}} \mathcal{V}^{+}$, and $k^{+}$is the dimension of the vector space $\pi^{+}(V)$.

Proof By Lemma 3.3, we have

$$
h(K, \Phi) \geq h\left(\pi^{+} K, \Phi^{+}\right) .
$$

Then, the result follows by applying Proposition 4.1 to $\pi^{+}(V)$. 


\section{An Upper Bound and Invariant Measures}

In this section, we establish an upper bound for the fiber entropy of subspaces and discuss the relation to the Morse spectrum.

First, we observe that there exists an invariant measure $\mu$ for the base flow $\theta$ on the compact metric space $B$. If $\mu$ is ergodic, the multiplicative ergodic theorem by Oseledets implies that there exist an invariant subset $B_{0} \subset B$ with $\mu\left(B_{0}\right)=1$, numbers $\lambda_{1}(\mu)<\ldots<\lambda_{r}(\mu), r=r(\mu)$, and a decomposition into measurable invariant subbundles, called Oseledets subbundles,

$$
\mathcal{V}_{b}=\mathcal{V}_{1, b}(\mu) \oplus \cdots \oplus \mathcal{V}_{r, b}(\mu),
$$

valid for every fiber $\mathcal{V}_{b}$ with $b \in B_{0}$, such that

$$
\lim _{t \rightarrow \pm \infty} \frac{1}{t} \log \|\Phi(t) v\|=\lambda_{i}(\mu) \text { iff } v \in \mathcal{V}_{i}(\mu) .
$$

Here, in every fiber, the convergence is uniform for $v$ with $\|v\|=1$; see for example, Arnold [2, Theorem 3.4.11], Johnson, Palmer, and Sell [12]. By [8, Corollary 5.5.17], each subbundle $\mathcal{V}_{i}(\mu)$ is contained in a Selgrade bundle $\mathcal{V}_{j}$ and

$$
\mathcal{V}_{j, b}=\sum \mathcal{V}_{i, b}(\mu) \text { and } \sum \lambda_{i}(\mu) \in \Sigma_{M o}\left(\mathcal{V}_{j}\right),
$$

where summation is over all $i$ with $\mathcal{V}_{i}(\mu) \subset \mathcal{V}_{j}$ and $b \in B_{0}$; the second equality follows, since $\sum \lambda_{i}(\mu)$ is the volume growth rate. An easy consequence of uniform convergence in (9) is that

$$
\lim _{t \rightarrow \pm \infty} \frac{1}{t} \log \|\Phi(t)\|=\lambda_{r}(\mu) .
$$

Next, we give a rough estimate for the topological entropy using Lyapunov exponents.

Proposition 5.1 Let $\Phi$ be a linear flow on a d-dimensional measurable vector bundle $\mathcal{V}$ with ergodic measure $\mu$ for the base flow $\theta$. With the notation from the multiplicative ergodic theorem, consider a $k$-dimensional parallelepiped $K \subset \mathcal{V}_{b}$, where $b \in B_{0}$. Then, the fiber entropy with respect to $K$ satisfies

$$
h(K ; \Phi) \leq k \cdot \lambda_{r}(\mu) .
$$

Proof Project $K$ to the corresponding Oseledets bundles $\mathcal{V}_{i}(\mu)$ with projections $p_{i}$ and denote the restricted flows by $\Phi_{i}$. Then, by Lemma 3.3(i),

$$
h(K ; \Phi) \leq h\left(p_{1} K ; \Phi_{1}\right)+\cdots+h\left(p_{r} K ; \Phi_{r}\right) .
$$

In a local chart, we may identify $K$ with a subset of $\mathbb{R}^{d}$ and hence there is $N \in \mathbb{N}$ with

$$
K \subset[-N, N]^{k} \text {. }
$$

For $\delta>0$ and $M:=\left\lceil\frac{1}{\delta}\right\rceil \in \mathbb{N}$, every point in $[-N, N]$ has distance less than $\frac{1}{M} \leq \delta$ to one of the $2 M N+1$ points in

$$
S:=\left\{x_{i}=\frac{i}{M}, i=-N, \ldots, N\right\} .
$$

Then, in the max-norm, every point in $K \subset[-N, N]^{k}$ has distance less than $\frac{1}{M} \leq \delta$ to one of the $(2 M N+1)^{k}$ points in the product $S^{k}$. Since

$$
\left\|\Phi_{t} x-\Phi_{t} y\right\| \leq\left\|\Phi_{t}\right\|\|x-y\|,
$$


we see that $S^{k}$ is an $\left(T, \delta \max _{t \in[0, T]}\left\|\Phi_{t}\right\|, K ; \Phi\right)$-spanning set of cardinality

$$
(2 M N+1)^{k} \leq M^{k}(2 N+1)^{k}=\left\lceil\frac{1}{\delta}\right\rceil^{k}(2 N+1)^{k} \leq\left(\frac{1}{\delta}+1\right)^{k}(2 N+1)^{k} .
$$

Thus for $\varepsilon>0$ and $\delta:=\varepsilon\left[\max _{t \in[0, T]}\left\|\Phi_{t}\right\|\right]^{-1}$, we find that the minimal cardinality of a $(T, \varepsilon, K ; \Phi)$-spanning set satisfies

$$
\begin{aligned}
r(T, \varepsilon, K ; \Phi) & \leq\left[\varepsilon^{-1} \max _{t \in[0, T]}\left\|\Phi_{t}\right\|+1\right]^{k}(2 N+1)^{k} \\
& =\left[\max _{t \in[0, T]}\left\|\Phi_{t}\right\|+\varepsilon\right]^{k} \varepsilon^{-k}(2 N+1)^{k}
\end{aligned}
$$

Up to a multiplicative constant, this estimate remains true for the norm on the vector bundle $\mathcal{V}$. Let

$$
\limsup _{T \rightarrow \infty} \frac{1}{T} \log r(T, \varepsilon, K ; \Phi)=\lim _{T_{i} \rightarrow \infty} \frac{1}{T_{i}} \log r\left(T_{i}, \varepsilon, K ; \Phi\right)
$$

There are $\tau_{i} \in\left[0, T_{i}\right]$ with $\left\|\Phi_{\tau_{i}}\right\|=\max _{t \in\left[0, T_{i}\right]}\left\|\Phi_{t}\right\|$. If $\tau_{i}$ and hence $\left\|\Phi_{\tau_{i}}\right\|$ remains bounded for $i \rightarrow \infty$, it is easy to see that $h(K, \Phi)=0$. Hence, we may assume that there is a subsequence of $\left(\tau_{i}\right)$, again denoted by $\left(\tau_{i}\right)$, with $\tau_{i} \rightarrow \infty$. It follows that

$$
\begin{aligned}
\limsup _{T \rightarrow \infty} \frac{1}{T} \log r(T, \varepsilon, K ; \Phi) & =\lim _{i \rightarrow \infty} \frac{1}{T_{i}} \log r\left(T_{i}, \varepsilon, K ; \Phi\right) \leq \lim _{i \rightarrow \infty} \frac{1}{\tau_{i}} \log r\left(T_{i}, \varepsilon, K ; \Phi\right) \\
& \leq \lim _{i \rightarrow \infty} \frac{1}{\tau_{i}}\left[\log \left(\left\|\Phi_{\tau_{i}}\right\|+\varepsilon\right)^{k}-k \log \varepsilon+k \log (2 N+1)\right] \\
& =k \lim _{i \rightarrow \infty} \frac{1}{\tau_{i}} \log \left\|\Phi_{\tau_{i}}\right\| \\
& \leq k \limsup \frac{1}{T} \log \left\|\Phi_{T}\right\| \\
& =k \cdot \lambda_{r}(\mu),
\end{aligned}
$$

where the last equality follows by (10).

Combining Proposition 5.1 with Theorem 4.4, we can prove the desired result on the relation between fiber entropy and the Morse spectrum.

Theorem 5.2 Consider a linear flow $\Phi$ on a vector bundle $\mathcal{V}$ with chain transitive base space $B$ and assume that $\Phi$ is hyperbolic, i.e., $0 \notin \Sigma_{M o}(\Phi)$.

(i) Then for every ergodic measure $\mu$ in $B$, there is a subset $B_{0}$ of full measure such that every $b \in B_{0}$ has the following property:

Let $V \subset \mathcal{V}_{b}$ be a $k$-dimensional subspace. The fiber entropy of $V$ satisfies

$$
h(V, \Phi) \in \Sigma_{M o}\left(\mathcal{M}_{k_{1}^{+}, \ldots, k_{l}^{+}}^{k^{+}}\right),
$$

where $\mathcal{M}_{k_{1}^{+}, \ldots, k_{l}^{+}}^{k^{+}}$is the Morse set that contains the omega limit of the point $\pi^{+}(V)$ for the induced flow on $\mathbb{G}_{k^{+}} \mathcal{V}^{+}$. 
(ii) Consider a Morse set $\mathcal{M}_{k_{1}^{+}, \ldots, k_{l}^{+}}^{k^{+}}$Then there is an ergodic measure $\mu^{*}$ on $B$ and a subset $B_{0}^{*}$ of full $\mu^{*}$-measure, such that for all $b \in B_{0}^{*}$ and every $k$-dimensional subspace $V \subset \mathcal{V}_{b} \cap \mathcal{M}_{k_{1}^{+}, \ldots, k_{l}^{+}}^{k^{+}}$

$$
h(V, \Phi)=\kappa^{*}\left(\Sigma_{M o}\left(\mathcal{M}_{k_{1}^{+}, \ldots, k_{l}^{+}}^{k^{+}}\right)\right) .
$$

An analogous property holds for $\kappa\left(\Sigma_{M o}\left(\mathcal{M}_{k_{1}^{+}, \ldots, k_{l}^{+}}^{k^{+}}\right)\right)$.

Proof (i) By Theorem 4.4, $h(V, \Phi)$ is greater than or equal to $\kappa^{*}\left(\mathcal{M}_{k_{1}^{+}, \ldots, k_{l}^{+}}^{k^{+}}\right)$. It remains to prove the other inequality, i.e., $h(V, \Phi) \leq \kappa\left(\mathcal{M}_{k_{1}^{+}, \ldots, k_{l}^{+}}^{k^{+}}\right)$. For every ergodic measure $\mu$ in the base space (there is at least one) and all points in an invariant subset of measure 1 , the assertions of the multiplicative ergodic theorem hold. Take $b \in B_{0}$ and consider the decomposition of $\mathcal{V}$ into the Selgrade bundles $\mathcal{V}_{j}$. By Lemma 3.2, we can consider the compact set $K$ as a $k$-parallelepiped spanned by vectors in $V$. Then $\pi_{j}^{+} K$ is a parallelepiped, and by Lemma 3.3, (ii) only the unstable Selgrade bundles have to be considered,

$$
h(V, \Phi)=h(K, \Phi)=h\left(\pi^{+} K, \Phi^{+}\right) \leq \sum h\left(\pi_{j}^{+} K, \Phi_{j}^{+}\right),
$$

where $\pi_{j}^{+}$denotes the projection to $\mathcal{V}_{j}^{+}$. Decompose $\mathcal{V}_{j}^{+}$into the Oseledets bundles $\mathcal{V}_{j, i}^{+}(\mu)$ with projections $\pi_{j, i}^{+}$and denote the corresponding restrictions of $\Phi$ by $\Phi_{j, i}^{+}$. We may assume that $\pi_{j, i}^{+} K$ is spanned by vectors $v_{i}, i=1, \ldots, k^{+}$in the Oseledets bundles. Lemma 3.3 (i) implies

$$
h\left(\pi_{j}^{+} K, \mathcal{V}_{j}^{+}\right) \leq \sum_{i} h\left(\pi_{j, i}^{+} K, \Phi_{j, i}^{+}\right)
$$

Now Proposition 5.1 applied to each $\Phi_{j, i}^{+}$yields

$$
h\left(\pi_{j, i}^{+} K ; \Phi_{j, i}^{+}\right) \leq k_{j, i}^{+} \cdot \lambda_{j, i}^{+}(\mu),
$$

where $\lambda_{j, i}^{+}(\mu)$ is the Lyapunov exponent of $\mathcal{V}_{j, i}^{+}(\mu)$ and $k_{j, i}^{+}=\operatorname{dim} \mathcal{V}_{j, i}^{+}(\mu)$. Then

$$
\begin{aligned}
h(V, \Phi) & \leq \sum_{j} h\left(\pi_{j}^{+} K, \Phi_{j}^{+}\right) \leq \sum_{j} \sum_{i} h\left(\pi_{j, i}^{+} K, \Phi_{j, i}^{+}\right) \\
& \leq \sum_{j} \sum_{i} k_{j, i}^{+} \cdot \lambda_{j, i}^{+}(\mu) \\
& =\limsup _{t \rightarrow \infty} \frac{1}{t} \log \left\|\Phi_{t} v_{1} \wedge \cdots \wedge \Phi_{t} v_{k^{+}}\right\|,
\end{aligned}
$$

since $\sum_{j} \sum_{i} k_{j, i}^{+} \cdot \lambda_{j, i}^{+}(\mu)$ is the volume growth rate of $\pi^{+} K$. Using again Lemma 2.5, we conclude that $h(V, \Phi) \leq \kappa\left(\mathcal{M}_{k_{1}^{+}, \ldots, k_{l}^{+}}^{k^{+}}\right)$as claimed.

(ii) By Theorem 2.4, there is an ergodic measure $\mu^{*}$ such that for all $b$ in a subset $B_{0}^{*}$ of full measure, one has

$$
\limsup _{t \rightarrow \infty} \frac{1}{t} \log \left\|\Phi_{t} v_{1} \wedge \cdots \wedge \Phi_{t} v_{k^{+}}\right\|=\min \Sigma_{M o}\left(\mathcal{M}_{k_{1}^{+}, \ldots, k_{l}^{+}}^{k^{+}}\right) .
$$


Then the arguments from (i) show that $h(V, \Phi)=\min \Sigma_{M o}\left(\mathcal{M}_{k_{1}^{+}, \ldots, k_{l}^{+}}^{k^{+}}\right)$. Similarly, the arguments in the proof of Theorem 4.4 show the assertion for $\max \Sigma_{M o}\left(\mathcal{M}_{k_{1}^{+}, \ldots, k_{l}^{+}}^{k^{+}}\right)$.

Remark 5.3 Theorem 5.2 with $k=d$ yields that $h_{\text {top }}(\Phi)$ is an element of the maximal Morse spectral interval in the Grassmann bundle $\mathbb{G}_{k^{+}} \mathcal{V}^{+}$with $k^{+}=\operatorname{dim} \mathcal{V}^{+}$. When the base space is a point, the flow reduces to a linear flow on a vector space, and its topological entropy is given by the sum of the unstable eigenvalues of the time-one map; cf. Walters [24, Theorem 8.14]. This, naturally, is the volume growth rate in the unstable subspace.

The results above apply, in particular, to bilinear control systems of the following form (cp. [6])

$$
\begin{aligned}
& \dot{x}=\left[A_{0}+\sum_{i=1}^{m} u_{i}(t) A_{i}\right] x(t) \text { in } \mathbb{R}^{d}, \\
& u \in \mathcal{U}=\left\{u: \mathbb{R} \rightarrow U \subset \mathbb{R}^{m}, \text { locally integrable }\right\}
\end{aligned}
$$

where $U \subset \mathbb{R}^{m}$ is compact and convex. Denote the solutions of the differential equation with initial condition $x(0)=x_{0} \in \mathbb{R}^{d}$ by $\varphi\left(t, x_{0}, u\right), t \in \mathbb{R}$.

Let $\theta_{t}: \mathcal{U} \rightarrow \mathcal{U}$ be the shift $\left(\theta_{t} u\right)(s)=u(t+s), s \in \mathbb{R}$, and endow $\mathcal{U} \subset L_{\infty}\left(\mathbb{R}, \mathbb{R}^{m}\right)=$ $\left(L_{1}(\mathbb{R}, \mathbb{R})\right)^{*}$ with the weak* topology. Then $\mathcal{U}$ becomes a compact metrizable space which is chain transitive. The flow defined by $\Phi_{t}(x, u)=\left(\varphi(t, x, u), \theta_{t} u\right)$ on $\mathcal{V}:=\mathbb{R}^{d} \times \mathcal{U} \rightarrow$ $\mathcal{U}$ is continuous and linear. The following result shows that only the fiber entropy gives interesting information on this flow, since already the base flow has infinite entropy.

Proposition 5.4 Under the assumptions above, the shift $\theta$ on $\mathcal{U}$ has infinite entropy, $h_{\text {top }}(\theta, \mathcal{U})=\infty$.

Proof We prove this by embedding, for every $N \in \mathbb{N}$, the shift on the symbol space on $N$ symbols into $\left(\theta_{1}, \mathcal{U}\right)$. Recall (see, e.g., Robinson [18], Section 2.5) that the shift $\sigma$ on the symbol space of maps from $\mathbb{Z}$ into $\{1, \ldots, N\}$ with metric

$$
\mathrm{d}(\mathbf{r}, \mathbf{s})=\sum_{k=-\infty}^{\infty} \frac{\delta\left(r_{k}, s_{k}\right)}{2^{k}}
$$

is a continuous map on the compact metric space $\Sigma_{N}=\{1, \ldots, N\}^{\mathbb{Z}}$. The entropy is $\log N$ by [18, Exercise 8.4]. Consider a set of $N$ elements $v_{1}, \ldots, v_{N} \in U$ and define a map

$$
f: \Sigma_{N} \rightarrow \mathcal{U}, f(\mathbf{r})=u_{\mathbf{r}}
$$

where the control $u_{\mathbf{r}}$ is given by

$$
u_{\mathbf{r}}(t)=v_{i} \text { for } t \in[i, i+1) \text { if } r_{i}=i .
$$

Clearly, the injective map $f$ conjugates the shifts $\sigma$ and $\theta_{1}, f \circ \sigma=\theta_{1} \circ f$. We will show that $f$ is continuous. Then, see [18, Theorem VIII.1.7], it follows that

$$
h_{\text {top }}\left(\theta_{1}, \mathcal{U}\right) \geq \log N \text { for every } N \in \mathbb{N}
$$


and the theorem is proved. In order to see continuity of $f$, consider $\mathbf{r} \in \Sigma_{N}$ and let $W$ be a neighborhood of $f(\mathbf{r})=u_{\mathbf{r}}$. Then there are $\varepsilon>0$ and $x_{1}, \ldots, x_{l} \in L_{1}\left(\mathbb{R}, \mathbb{R}^{m}\right)$ with

$$
W_{0}=\left\{u \in L_{\infty}\left(\mathbb{R}, \mathbb{R}^{m}\right), \quad \begin{array}{c}
\left|\int_{\mathbb{R}}\left\langle u_{\mathbf{r}}(t)-u(t), x_{j}(t)\right\rangle d t\right|<\varepsilon \text { for } j=1, \ldots, l \\
\text { and } u(t) \in U \text { for almost all } t \in \mathbb{R}
\end{array}\right\} \subset W,
$$

because the sets of this form are a subbasis of the weak* topology (see, e.g., [25, p. 31] or [10]). Because $x_{j} \in L_{1}\left(\mathbb{R}, \mathbb{R}^{m}\right)$, there is $K \in \mathbb{N}$ such that for $j=1, \ldots, l$

$$
\int_{\mathbb{R} \backslash[-K, K]}\left|x_{j}(t)\right| d t<\frac{\varepsilon}{\operatorname{diam} U},
$$

where $\operatorname{diam} U=\sup \left\{\left|u_{1}-u_{2}\right|, u_{1}, u_{2} \in U\right\}$. There is $\delta>0$ such that for all $\mathbf{s} \in \Sigma_{N}$

$$
\mathrm{d}(\mathbf{r}, \mathbf{s})=\sum_{k=-\infty}^{\infty} \frac{\delta\left(r_{k}, s_{k}\right)}{2^{k}}<\delta
$$

implies $r_{k}=s_{k}$ for all $k \leq K$. Hence for all $\mathbf{s}$ with $\mathrm{d}(\mathbf{r}, \mathbf{s})<\delta$, it follows that

$$
\begin{aligned}
& \left|\int_{\mathbb{R}}\left\langle u_{\mathbf{r}}(t)-u_{\mathbf{s}}(t), x_{j}(t)\right\rangle d t\right| \\
& \leq\left|\int_{-K}^{K}\left\langle u_{\mathbf{r}}(t)-u_{\mathbf{s}}(t), x_{j}(t)\right\rangle d t\right|+\left|\int_{\mathbb{R} \backslash[-T, T]}\left\langle u_{\mathbf{r}}(t)-u_{\mathbf{s}}(t), x_{j}(t)\right\rangle d t\right| \leq \varepsilon,
\end{aligned}
$$

and hence $f(\mathbf{s}) \in W_{0} \subset W$ as desired.

Acknowledgments F. Colonius is supported by DFG grant Co 124/17-2 within DFG Priority Program 1305 Control Theory of Digitally Networked Dynamical Systems. L.A.B. San Martin is supported by CNPq grant $\mathrm{n}^{\mathrm{o}} 303755 / 2009-1$ and FAPESP grant $\mathrm{n}^{\mathrm{o}}$ 07/06896-5. A.J. da Silva is supported by CAPES grant $\mathrm{n}^{\mathrm{o}}$ 4229/10-0.

\section{References}

1. Alongi J, Nelson G. Recurrence and topology. Am Math Soc. 2007.

2. Arnold L. Random dynamical systems. Springer-Verlag; 1998.

3. Bogenschütz T. Entropy, pressure and a variational principle for random dynamical systems. Random and Computational Dynamics Unknown Month 1992;1(1):99-116.

4. Bowen R. Entropy for group endomorphisms and homogeneous spaces. Trans Am Math Soc. 1971; 153. Erratum, 1973;181: 509-510.

5. Braga Barros C, San Martin LAB. Chain transitive sets for flows on flag bundles. Forum Math. 2007;19:19-60.

6. Colonius F, Fabbri R, Johnson R. Chain recurrence, growth rates and ergodic limits. Ergodic Theory and Dynamical Systems 2004;27:1509-1524.

7. Colonius F, Kliemann W. The Morse spectrum of linear flows on vector bundles. Trans Am Math Soc. 1996;348:4355-4388.

8. Colonius F, Kliemann W. The dynamics of control. Boston: Birkhäuser; 2000.

9. Colonius F, Kliemann W. Morse decompositions and spectra on flag bundles. J Dyn Diff Equat. 2002;14:719-741.

10. Dunford N, Schwartz JT. Linear operators, Part I: general theory. Wiley-Interscience; 1977.

11. Handel M, Kitchens B. Metrics and entropy for non-compact spaces. Israel J Math. 1995;91:253-271.

12. Johnson RA, Palmer KJ, Sell GR. Ergodic properties of linear dynamical systems. SIAM J Math Anal. 1987;18:1-33.

13. Karoubi M. K-Theory, an introduction. Springer-Verlag; 1978.

14. Kolyada S, Snoha L. Topological entropy for nonautonomous dynamical systems. Random and Comput Dyn. 1996;4:205-233. 
15. Patrão M. Entropy and its variational principle for non-compact metric spaces. Ergodic Theor Dyn Syst. 2010;30:1529-1542.

16. Patrão M, San Martin LAB. Chain recurrence of flows and semiflows on fiber bundles. Discrete Contin Dynam Syst A. 2007; 17:113-139.

17. Petersen K. Ergodic theory. Cambridge University Press; 1989.

18. Robinson C. Dynamical systems. Stability, symbolic dynamics, and chaos. CRC Press; 1995.

19. Sacker RJ, Sell GR. A spectral theory for linear differential systems. J Diff Equ. 1978;27:320-358.

20. Salamon D, Zehnder E. Flows on vector bundles and hyperbolic sets. Trans Amer Math Soc. 1988;306:623-649.

21. San Martin LAB, Seco L. Morse and Lyapunov spectra and dynamics on flag bundles. Ergodic Theor Dyn Syst. 2009;30:893-922.

22. Selgrade J. Isolated invariant sets for flows on vector bundles. Trans Amer Math Soc. 1975;203:259-390.

23. Zhang J-L, Chen L-X. Lower bounds of the topological entropy for nonautonomous dynamical systems. Appl Math J Chinese Univ. 2009;24(1):76-82.

24. Walters P. An introduction to Ergodic theory. Springer-Verlag; 1982.

25. Warga J. Optimal control of differential and functional equations. Academic; 1972. 